\title{
Coexistence of electron whispering-gallery modes and atomic collapse states in graphene/WSe2 heterostructure quantum dots
}

\section{Qi Zheng}

Beijing Normal University

\section{Yu-Chen Zhuang}

Peking University

Qingfeng Sun

Peking University

Lin He ( $\nabla$ helin@bnu.edu.cn )

Beijing Normal University https://orcid.org/0000-0001-5251-1687

\section{Article}

Keywords: electron whispering-gallery modes, atomic collapse states, graphene/WSe2 heterostructure quantum dots

Posted Date: August 13th, 2021

DOl: https://doi.org/10.21203/rs.3.rs-763571/v1

License: (c) (i) This work is licensed under a Creative Commons Attribution 4.0 International License. Read Full License

Version of Record: A version of this preprint was published at Nature Communications on March 24th, 2022. See the published version at https://doi.org/10.1038/s41467-022-29251-2. 


\section{Title: Coexistence of electron whispering-gallery modes and atomic collapse}

\section{states in graphene/WSe 2 heterostructure quantum dots}

Authors: Qi Zheng ${ }^{1, \S}$, Yu-Chen Zhuang ${ }^{2, \S}$, Qing-Feng Sun ${ }^{2,3,4, \dagger}$, Lin $\mathrm{He}^{1, \dagger}$

\section{Affiliations:}

$5{ }^{1}$ Center for Advanced Quantum Studies, Department of Physics, Beijing Normal University, Beijing, 100875, People's Republic of China

${ }^{2}$ International Center for Quantum Materials, School of Physics, Peking University, Beijing, 100871, China

${ }^{3}$ Collaborative Innovation Center of Quantum Matter, Beijing 100871, China

$10{ }^{4}$ Beijing Academy of Quantum Information Sciences, West Bld. \#3, No. 10 Xibeiwang East Road, Haidian District, Beijing 100193, China

$\S$ These authors contributed equally to this work.

${ }^{\dagger}$ Correspondence and requests for materials should be addressed to Qing-Feng Sun (email:

15 sunqf@pku.edu.cn) and Lin He (e-mail: helin@bnu.edu.cn).

The relativistic massless charge carriers with a Fermi velocity of about $c / 300$ in graphene enable us to realize two distinct types of resonances ( $c$, the speed of light in vacuum). One is electron whispering-gallery mode in graphene quantum dots arising from the Klein tunneling of the massless Dirac fermions. The other is atomic collapse state, which has never

20 been observed in experiment with real atoms due to the difficulty of producing heavy nuclei with charge $Z>170$, however, can be realized near a Coulomb impurity in graphene with a charge $Z \geq 1$ because of the "small" velocity of the Dirac excitations. Here, unexpectedly, we demonstrate that both the electron whispering-gallery modes and atomic collapse states coexist in graphene/WSe 2 heterostructure quantum dots due to the Coulomb-like potential

25 near their edges. By applying a perpendicular magnetic field, evolution from the atomic collapse states to unusual Landau levels in the collapse regime are explored for the first time. 
Many exotic electronic properties of graphene are rooted in its relativistic massless charge carriers (1-4). For example, the massless Dirac fermions nature of the charge carriers in graphene enables us to demonstrate several oddball predictions by quantum electrodynamics (QED), among which the Klein tunneling (5) and atomic collapse (6-8) are the two most famous effects that have

5 attracted much attention. Very recently, it was demonstrated that the two effects lead to the formation of two types of quasibound states in graphene (9-18). The Klein tunneling, i.e., the anisotropic transmission of the massless Dirac fermions across the potential barrier, in graphene leads to the formation of quasibound states in circular $p$ - $n$ junctions, i.e., graphene quantum dots (GQDs), via whispering-gallery modes (WGMs) (9-15). Because of the "small" velocity of the

10 Dirac fermions, a Coulomb impurity in graphene with a charge $\mathrm{Z} \geq 1$ can result in the formation of atomic collapse states (ACSs) around it $(16,17)$. In previous experiments, pronounced resonances of the two types of the quasibound states were clearly observed (9-18). Due to their distinct underlying origins, the two quasibound states are expected to be observed in the two different systems.

15 Here, we demonstrate the coexistence of the electron WGMs and ACSs in graphene/WSe 2 heterostructure QDs. Because of the Coulomb-like potential near the edges of the QDs, we observe WGMs near the edge and ACSs in the center of the graphene/WSe 2 heterostructure QDs. Moreover, the ACSs are further explored in the presence of high magnetic fields. The study about effect of magnetic fields on the ACSs has a long history (19). However, such a longstanding prediction remains highly controversial because that the theoretical results are contradictory (20-25) and, 
more importantly, an experimental verification of this fundamental prediction is still lacking up to now $(25,26)$. Our experiments demonstrate that the ACSs still exist in magnetic fields and evolution from the ACSs to unusual Landau levels in the collapse regime are measured.

The graphene/ $\mathrm{WSe}_{2}$ heterostructure was obtained by using a wet transfer fabrication of a 5 monolayer graphene on mechanical-exfoliated thick $\mathrm{WSe}_{2}$ sheets (see methods for details of the sample preparation). In our experiment, nanoscale $\mathrm{WSe}_{2}$ QDs were surprisingly observed on surface of freshly mechanical exfoliated $\mathrm{WSe}_{2}$ sheets, as shown in atomic force microscopy (AFM) image of Fig. 1a. Usually, the thickness of the $\mathrm{WSe}_{2} \mathrm{QDs}$ is the same as a $\mathrm{WSe}_{2}$ monolayer and the diameter is less than $10 \mathrm{~nm}$. Such $\mathrm{WSe}_{2}$ QDs also can be observed when mechanical-exfoliated 10 thick $\mathrm{WSe}_{2}$ is covered with graphene monolayer, i.e., in the graphene/ $\mathrm{WSe}_{2}$ heterostructure, as shown in scanning tunneling microscope (STM) image of Fig. 1b (also see Fig. S1 for AFM images of the graphene/ $\mathrm{WSe}_{2}$ heterostructure). At present, the exact origin for the emergence of the nanoscale $\mathrm{WSe}_{2} \mathrm{QDs}$ is unclear. In our experiment, nanoscale monolayer-thick $\mathrm{WSe}_{2}$ anti-dots are usually observed around the $\mathrm{WSe}_{2}$ QDs (See Fig. S2), which suggests that the $\mathrm{WSe}_{2}$ QDs are

15 generated from the anti-dots during the process of mechanical exfoliation. Figure 1c shows an atomic-resolved STM image around a graphene/ $\mathrm{WSe}_{2}$ heterostructure QD. No atomic defect and strain structure can be detected in graphene above the $\mathrm{WSe}_{2} \mathrm{QD}$. Fast Fourier transform (FFT) images inside and outside the QD are identical (see inset of Fig. 1c and fig. S2b) and the relative rotation angle between graphene and $\mathrm{WSe}_{2}$ is measured as about $20.4^{\circ}$ (the rotation angle between the $\mathrm{WSe}_{2} \mathrm{QD}$ and the $\mathrm{WSe}_{2}$ substrate is zero). A schematic side view of the graphene/ $\mathrm{WSe}_{2}$ 
heterostructure is shown in Fig. $1 \mathrm{~d}$.

It is interesting to find that the $\mathrm{WSe}_{2} \mathrm{QD}$ strongly modifies electronic properties of the graphene above it. Figure 1e shows two representative scanning tunneling spectroscopy (STS) spectra of the graphene on and off the $\mathrm{WSe}_{2} \mathrm{QD}$. The STS, i.e., $\mathrm{d} I / \mathrm{d} V$, measurement of the graphene off the

$5 \mathrm{WSe}_{2}$ QD shows a typical V-shaped spectrum profile of graphene with the Dirac point at $E_{D}^{\text {Off }} \approx-0.2 \mathrm{eV}$ (p-doping). Whereas, spectrum of graphene on the $\mathrm{WSe}_{2} \mathrm{QD}$ displays a series of resonance peaks with the Dirac point estimated as $E_{D}^{O n} \approx 0.3 \mathrm{eV}$ (n-doping). Obviously, the WSe 2 QD generates a circular p-n junction, i.e., a GQD, on graphene. The almost equally spaced peaks in the spectrum are the quasibound states confined in the GQD via the WGMs (9-15). Such a result

10 is further confirmed by carrying out STS mapping at different resonance energies (Fig. 1f and Fig. S3). For the WGMs, the quasibound states, except the lowest one, display shell structures and are progressively closer to the GQD edge with increasing the energy (Fig. S7), as observed in our experiment. According to our experiment, the potential difference $(\Delta U)$ on and off the GQDs shows positive correlation to the ratio $(\eta)$ of the number of edge atoms to the number of inner 15 atoms in the $\mathrm{WSe}_{2} \mathrm{QD}$ (see Fig. S4). Therefore, the large potential difference on and off the GQD may arise from the edge of the $\mathrm{WSe}_{2} \mathrm{QD}$. The dangling bonds at the edge can significantly change the electronic property and work function of the $\mathrm{WSe}_{2} \mathrm{QD}(27-29)$, generating the large circular electrostatic potential on graphene covering it.

To further explore electronic properties of the GQDs, we performed the radially $\mathrm{d} I / \mathrm{d} V$ 
spectroscopic maps of several GQDs with different sizes and potentials, as shown in Fig. 2 (see insets of Fig. S5 for the STM images of the GQDs). By following the spatial dependence of global local density of states (LDOS) in the maps, it is interesting to note that the $\mathrm{WSe}_{2}$ QDs generate a Coulomb-like electrostatic potential: $V_{\beta}(r)=\left\{\begin{array}{ll}\hbar v_{F} \frac{\beta}{r_{0}}, & r \leq r_{0} \\ \hbar v_{F} \frac{\beta}{r}, & r>r_{0}\end{array}\right.$, where $\hbar$ is the reduced Planck 5 constant, $v_{F}$ is the Fermi velocity, $r$ is the distance from the center of GQD, $\beta=Z \alpha$ with $\alpha \sim 2.5$ the fine structure constant of graphene (8), and $r_{0}$ is the cutoff radius of Coulomb potential. We obtained different values of $\beta$ and $r_{0}$ for different GQDs. As shown in Fig. 2, the GQDs with different $\beta$ exhibit quite different features of the quasibound states. For the $\beta=2 \mathrm{GQD}$, there is only one resonance peak at the center of the GQD (Fig. 2a, Top). Whereas, for the $\beta=4.3 \mathrm{GQD}$, besides several quasibound states confined via the WGMs at the edge of the GQD, there are three unequally spaced resonance peaks located at the center of the GQD (Fig. 2c, Top panels). To fully understand these unusual quasibound states, we numerically solved the problem for a Coulomblike electrostatic potential with different values of $\beta$ and $r_{0}$ in the graphene monolayer (the values of $\beta$ and $r_{0}$ are extracted from our experimental results) (see supplementary information for the 15 details). Bottom panels of Fig. 2 show the theoretical space-energy maps of the LDOS of the GQDs, which are in good agreement with the experimental results (see Fig. S5 for $\mathrm{d} I / \mathrm{d} V$ spectra and corresponding simulated LDOS at different positions of the GQDs, see Fig. S7 for the spatial distribution of the quasibound states). According to our analysis, the resonance peaks located at the edge of GQDs arise from the quasibound states via the WGMs confinement. The energy levels 
of these quasibound states at the edge can be estimated as $\hbar v_{F} / R_{\text {eff }}$ (here $R_{\text {eff }}$ is the effective radius of the GQD), as observed in our experiment (see Fig. S4d) and reported in previous studies $(10,12,13)$. Whereas the energy levels of the quasibound states at the center of the GQD follow an exponential function $E_{n}=\frac{\hbar v_{F} \beta}{r_{0}} e^{-\frac{\pi}{\gamma} n}+E_{D}$, where $\gamma=\sqrt{\beta^{2}-(m+1 / 2)^{2}}, E_{D}$ is the energy of

5 Dirac point $[m$ denotes the orbital states $(m=0, \pm 1, \pm 2, \ldots)]$. This is a characteristic feature of the ACSs in the supercritical regime due to the Coulomb-like electrostatic potential (see Fig. S8 and the details of discuss) (6-8). Therefore, our experimental results, supported by our theoretical calculation, strongly indicate the coexistence of the WGMs and ACSs in the graphene/ $\mathrm{WSe}_{2}$ heterostructure GQDs. In previous studies of the Coulomb impurity in graphene with a 10 supercritical charge, only the ACSs are observed because of the small $r_{0} \sim 0.5 \mathrm{~nm}(16,17)$. In this work, the Coulomb-like potential near the edges of the GQDs and the increase of about one order of magnitude of the $r_{0}$ allow us to observe both the WGMs and the ACSs.

The Coulomb-like potential also strongly affects the electronic properties of the GQDs in the presence of magnetic fields. By applying a perpendicular magnetic field, we can observe well 15 defined Landau levels (LLs) of massless Dirac fermions at positions away from the GQD (see Fig. S9a). When approaching the GQD, the Coulomb-like potential generates pronounced bending of the LLs (see Fig. S9a for the experimental result and theoretical simulation). Figure 3 shows radially spectroscopic maps around the $\beta=2.4 \mathrm{GQD}$ in three different magnetic fields. Near the edge of the GQD, the bending of the LLs follows the Coulomb-like electrostatic potential. Inside 
the GQD, complex evolution of LDOS due to the transition from the confinement of the electrostatic potential to confinement of magnetic field is observed with increasing the magnetic field (see Fig. S10 for more experimental data). At $B=10 \mathrm{~T}$, we can observe LLs inside the GQD. However, the $N=-1$ LL is split into three peaks: two of them with higher energies are localized 5 in the center of the GQD and the third one is mainly located at the edge of the GQD. The splitting does not occur in pairs and the energy spacing of the splitting is as large as $\sim 40 \mathrm{meV}$ (Fig. S11), which removes valley and spin splitting as the origin of the observed phenomenon. The splitting LLs should be attributed to lifting the orbital degeneracy of LLs, which can be understood by considering the quantum-mechanical electron motion in the presence of a magnetic field and a

10 Coulomb-like electrostatic potential. Considering the effect of the magnetic field and the electrostatic potential, the equation thus reads:

$$
\left[v_{F} \vec{\sigma} \cdot(-i \hbar \vec{\nabla}+e \vec{A})+V_{\beta}(\vec{r})\right] \psi(\vec{r})=E \psi(\vec{r}),
$$

where $\vec{\sigma}=\left(\sigma_{x}, \sigma_{y}\right)$ are the Pauli matrices, $\vec{A}=(\vec{B} \times \vec{r}) / 2$ is the vector potential $(21,25,26), e$ is the electron charge. Due to the axial symmetry of the electrostatic potential in the GQD, we can

15 describe the eigenstates by the orbital quantum number $m$ (here, we neglect spin). In the absence of the GQD, the eigen-energies $E_{N m}$ have infinite orbital degeneracy $\left[\psi_{N m}(\vec{r})\right.$ where $\left.m \geq-|N|\right]$ independent of $m$ because of translational invariance. The GQD lifts this orbital degeneracy $m$ and the LLs are split into a series of sublevels, which exhibit similar behavior as that observed around charged impurities $(17,26)$, due to the Coulomb-like electrostatic potential. However, previous experiments $(17,26)$ in the presence of a magnetic field were limited to a charge impurity in the 
subcritical regime. Further, the small cutoff radius of a charge impurity prohibits to explore the evolution from the ACSs to the LLs in experiment. Such difficulties can be naturally overcome in the studied GQDs.

The detailed comparison between experiment and theory can be made by numerically solving

5 the problem for two dimensional massless Dirac fermion of graphene monolayer in the presence of Coulomb-like electrostatic potential $V_{\beta}(\vec{r})$ and a magnetic field $B$ (see supplementary information for the details). The calculated radially LDOS maps in the different magnetic fields display that the orbital degeneracy is lifted, which is well consistent with our experimental results (Fig. 3). Based on the calculated results, we can identify the orbital states of the split -1 LL (Marked

10 in Fig. 3). Thanks to the high-quality LLs in the GQD, $m=-1$ orbital state of -1 LL can be clearly identified and exhibits some characteristics distinguished from that observed in the subcritical regime $(17,26)$. The most important feature is that the $m=-1$ orbital state can be viewed as the evolution of the ACS with increasing magnetic field. At zero magnetic field, the broad ACS is located at the center of the GQD and, interestingly, the narrower $m=-1$ orbital state appears in the 15 same energy region in the presence of high magnetic field. Such a result indicates directly connection of the ACS and the lowest orbital state $(m=-1)$ of the -1 LL.

To better explore the evolution of the ACS in the presence of magnetic fields, we summarize the measured LLs at the center of the $\beta=2.4 \mathrm{GQD}$ as a function of the square root of the magnetic field $\sqrt{B}$ (red dots in Fig. 4, see Fig. S12 for the corresponding STS spectra). The evolution of 
LLs displayed a nonlinear dependence on the square root of the magnetic field, which is quite different from the feature of pristine graphene monolayer under magnetic field. The theoretical map of LDOS at the center of the $\beta=2.4$ GQD is also plotted as a function of $\sqrt{B}$ (see supplementary information for the details), as shown in Fig. 4. With increasing magnetic field, the

5 perturbed LLs $(N=0, N=-1, N=-2)$ display nonlinear dependence on $\sqrt{B}$. At a higher $\sqrt{B}$, the -1 LL and -2 LL are well distinctive, which split into low-energy orbital states ( $m=-1, m=$ 0 ). However, we did not observe the splitting of the -2 LL in the experiment, which is probably due to the large full width at half maximum (FWHM) of the LL peaks, prohibiting the observation of the splitting in the experiment. Furthermore, the ACS-R1 resonance is obvious at lower $\sqrt{B}$, 10 and is well connected to the $m=-1$ orbital state of -1 LL. Similarly, ACS-R2 resonance has the similar characteristic, connected to the $m=-1$ orbital state of -2 LL. However, such a feature is harder to be recognized in the experiment due to the broadening peak of the -2 LL. Our experiments, complemented by theoretical calculations, explicitly demonstrated the existence of ACSs in the presence of high magnetic fields and revealed the close connection between the ACS and the 15 lowest orbital state $(m=-1)$ of the LLs.

\section{References}

1. Castro Neto, A. H., Peres, N. M. R., Novoselov, K. S. \& Geim, A. K. The electronic properties of graphene. Rev. Mod. Phys. 81, 109-162 (2009).

2. Vozmediano, M. A. H., Katsnelson, M. I. \& Guinea, F. Gauge fields in graphene. Physics Rep. 496, 109-148 (2010). 
3. Das Sarma, S., Adam, S., Hwang, E. \& Rossi, E. Electronic transport in two-dimensional graphene. Rev. Mod. Phys. 83, 407-470 (2011).

4. Goerbig, M. O. Electronic properties of graphene in a strong magnetic field. Rev. Mod. Phys. 83, 1193-1243 (2011).

5 5. Katsnelson, M. I., Novoselov, K. S. \& Geim, A. K. Chiral tunnelling and the Klein paradox in graphene. Nat. Phys. 2, 620-625 (2006).

6. Pereira, V. M., Nilsson, J. \& Castro Neto, A. H. Coulomb impurity problem in graphene. Phys. Rev. Lett. 99, 166802 (2007).

7. Shytov, A. V., Katsnelson, M. I. \& Levitov, L. S. Vacuum Polarization and Screening of Supercritical Impurities in Graphene. Phys. Rev. Lett. 99, 236801 (2007).

8. Shytov, A. V., Katsnelson, M. I. \& Levitov, L. S. Atomic collapse and quasi-rydberg states in graphene. Phys. Rev. Lett. 99, 246802 (2007).

9. Zhao, Y. et al. Creating and probing electron whispering-gallery modes in graphene. Science 348, 672-675 (2015).

15 10. Gutiérrez, C., Brown, L., Kim, C.-J., Park, J. \& Pasupathy, A. N. Klein tunnelling and electron trapping in nanometre-scale graphene quantum dots. Nat. Phys. 12, 1069-1075 (2016).

11. Lee, J. et al. Imaging electrostatically confined Dirac fermions in graphene quantum dots. Nat. Phys. 12, 1032-1036 (2016).

12. Bai, K.-K. et al. Generating nanoscale and atomically-sharp p-n junctions in graphene via monolayer-vacancy-island engineering of Cu surface. Phys. Rev. B 97, 045413 (2018). 
13. Fu, Z. Q., Bai, K. K., Ren, Y. N., Zhou, J. J. \& He, L. Coulomb interaction in quasibound states of graphene quantum dots. Phys. Rev. B 101, 235310 (2020).

14. Fu, Z.-Q. et al. Relativistic Artificial Molecules Realized by Two Coupled Graphene Quantum Dots. Nano Lett. 20, 6738 (2020).

5 15. Ghahari, F. et al. An on/off Berry phase switch in circular graphene resonators. Science 356, 845 (2017).

16. Wang, Y. et al. Observing atomic collapse resonances in artificial nuclei on graphene. Science 340, 734-737 (2013).

17. Mao, J. et al. Realization of a tunable artificial atom at a supercritically charged vacancy in graphene. Nat. Phys. 12, 545-549 (2016).

18. Jiang, Y. et al. Tuning a circular $\mathrm{p}-\mathrm{n}$ junction in graphene from quantum confinement to optical guiding. Nat. Nanotechnol. 12, 1045-1049 (2017).

19. Reinhardt, J. \& Greiner, W. Quantum electrodynamics of strong fields. Rep. Prog. Phys. 40, 219-295 (1977).

15 20. Gamayun, O. V., Gorbar, E. V. \& Gusynin, V. P. Magnetic field driven instability of a charged center in graphene. Phys. Rev. B 83, 235104 (2011).

21. Sobol, O. O., Pyatkovskiy, P. K., Gorbar, E. V. \& Gusynin, V. P. Screening of a charged impurity in graphene in a magnetic field. Phys. Rev. B 94, 115409 (2016).

22. Zhang, Y., Barlas, Y. \& Yang, K. Coulomb impurity under magnetic field in graphene: A semiclassical approach. Phys. Rev. B 85, 165423 (2012). 
23. Maier, T. \& Siedentop, H. Stability of impurities with Coulomb potential in graphene with homogeneous magnetic field. J. Math. Phys. 53, 095207 (2012).

24. Kim, S. C. \& Eric Yang, S. R. Coulomb impurity problem of graphene in magnetic fields. Ann. Phys. 347, 21-31 (2014).

25. Moldovan, D., Masir, M. R. \& Peeters, F. M. Magnetic field dependence of the atomic collapse state in graphene. 2D Mater. 5, 015017 (2018).

26. Luican-Mayer, A. et al. Screening charged impurities and lifting the orbital degeneracy in graphene by populating landau levels. Phys. Rev. Lett. 112, 036804 (2014).

27. Zhang, Y. et al. Electronic Structure, Surface Doping, and Optical Response in Epitaxial $\mathrm{WSe}_{2}$ Thin Films. Nano Lett. 16, 2485-2491 (2016).

28. Addou, R. \& Wallace, R. M. Surface Analysis of $\mathrm{WSe}_{2}$ Crystals: Spatial and Electronic Variability. ACS Appl. Mater. Interfaces 8, 26400-26406 (2016).

29. Kahn, A. Fermi level, work function and vacuum level. Mater. Horiz. 3, 7-10 (2016).

\section{Acknowledgments:}

15 This work was supported by the National Natural Science Foundation of China (Grant Nos.

11974050, 11674029, 11921005) and National Key R and D Program of China (Grant No.

2017YFA0303301). L.H. also acknowledges support from the National Program for Support of

Top-notch Young Professionals, support from "the Fundamental Research Funds for the Central Universities", and support from "Chang Jiang Scholars Program". 


\section{Author contributions}

Q.Z. performed the sample synthesis, characterization and STM/STS measurements. Q.Z., Y.C.Z., and L.H. analyzed the data. Y.C.Z. carried out the theoretical calculations. L.H. conceived and

5 provided advice on the experiment and analysis. Q.F.S. conceived and provided advice on the theoretical calculations. Q.Z. and L.H. wrote the paper with the input from others. All authors participated in the data discussion.

\section{Methods}

10 CVD Growth of Graphene. The large area graphene monolayer films were grown on a $20 \times 20 \mathrm{~mm}^{2}$ polycrystalline copper $(\mathrm{Cu})$ foil (Alfa Aesar, $99.8 \%$ purity, $25 \mu \mathrm{m}$ thick) via a low pressure chemical vapor deposition (LPCVD) method. The cleaned $\mathrm{Cu}$ foil was loaded into one quartz boat in center of the tube furnace. Ar flow of $50 \mathrm{sccm}$ (Standard Cubic Centimeter per Minutes) and $\mathrm{H}_{2}$ flow of $50 \mathrm{sccm}$ were maintained throughout the whole growth process. The $\mathrm{Cu}$

15 foil was heated from room temperature to $1030{ }^{\circ} \mathrm{C}$ in $30 \mathrm{~min}$ and annealed at $1030{ }^{\circ} \mathrm{C}$ for six hours. Then $\mathrm{CH}_{4}$ flow of $5 \mathrm{sccm}$ was introduced for 20 min to grow high-quality large area graphene monolayer. Finally, the furnace was cooled down naturally to room temperature.

Construction of graphene/WSe 2 heterostructure. We used conventional wet etching technique with polymethyl methacrylate (PMMA) to transfer graphene monolayer onto the substrate. PMMA

20 was first uniformly coated on $\mathrm{Cu}$ foil with graphene monolayer. We transferred the $\mathrm{Cu} /$ graphene/PMMA film into ammonium persulfate solution, and then the underlying $\mathrm{Cu}$ foil was 
etched away. The graphene/PMMA film was cleaned in deionized water for hours. The $\mathrm{WSe}_{2}$ crystal was separated into thick-layer $\mathrm{WSe}_{2}$ sheets by traditional mechanical exfoliation technology and then transferred to $8 \times 8 \mathrm{~mm}^{2}$ highly N-doped Si wafer [(100) oriented, $500 \mu \mathrm{m}$ thick]. We placed graphene/PMMA onto Si wafer which has been transferred with $\mathrm{WSe}_{2}$ sheets in

5 advance. Finally, the PMMA was removed by acetone and then annealed in low pressure with Ar flow of $50 \mathrm{sccm}$ and $\mathrm{H}_{2}$ flow of $50 \mathrm{sccm}$ at $\sim 300{ }^{\circ} \mathrm{C}$ for 1 hours.

AFM, STM and STS Measurements. The topographical images are measured by atomic force microscope (AFM, Bruker Multimode 8) with a tapping mode. We employed the n-doped Si tip coated with Platinum-Iridium (Bruker, SCM-PIT-V2, frequency 50-100KHz, spring constant 1.5-

$106 \mathrm{~N} / \mathrm{m}$ ) to characterize $\mathrm{WSe}_{2}$ and graphene/WSe $\mathrm{W}_{2}$ heterostructure samples. STM/STS measurements were performed in low-temperature (77 K for Fig. S5a and c, $4.2 \mathrm{~K}$ for Fig. S5b) and ultrahigh-vacuum ( $10^{-10}$ Torr) scanning probe microscopes [USM-1400 (77 K) and USM$1300(4.2 \mathrm{~K})$ ] from UNISOKU. The tips were obtained by chemical etching from a Pt/Ir (80:20\%) alloy wire. The differential conductance $(\mathrm{d} / / \mathrm{d} V)$ measurements were taken by a standard lock-in 15 technique with an ac bias modulation of $5 \mathrm{mV}$ and $793 \mathrm{~Hz}$ signal added to the tunneling bias. 
a
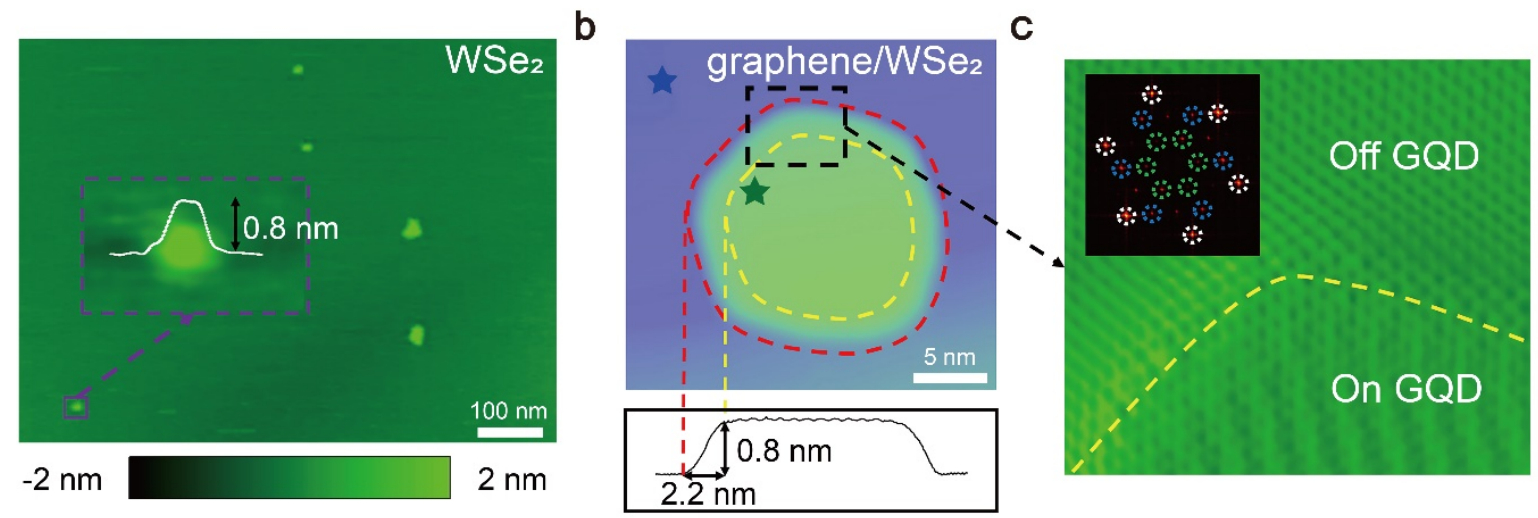

d

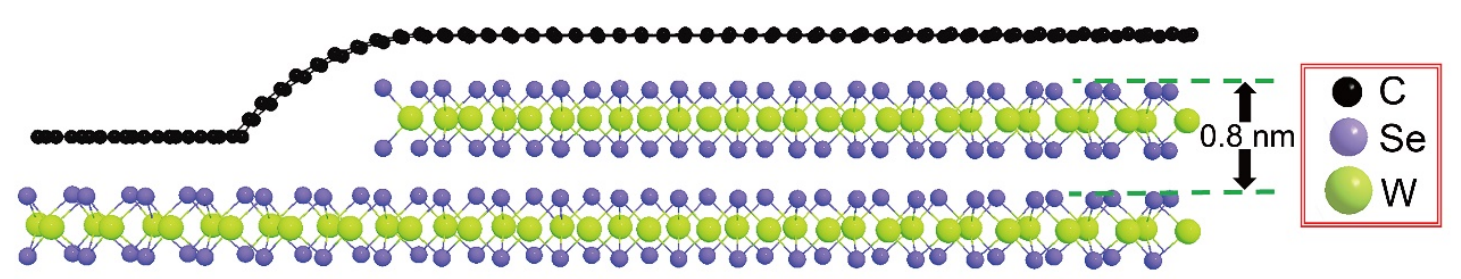

e

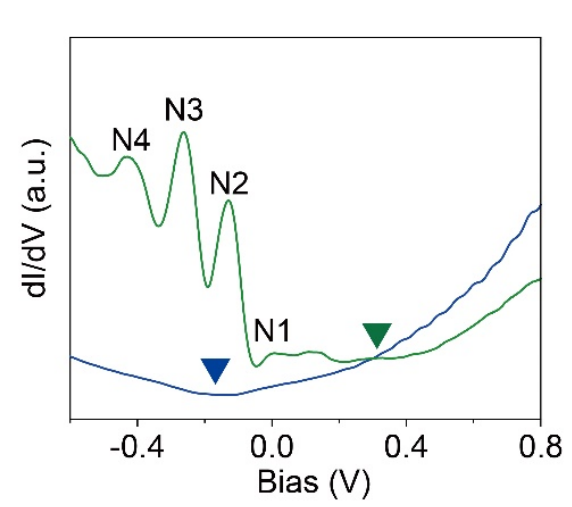

\section{f}

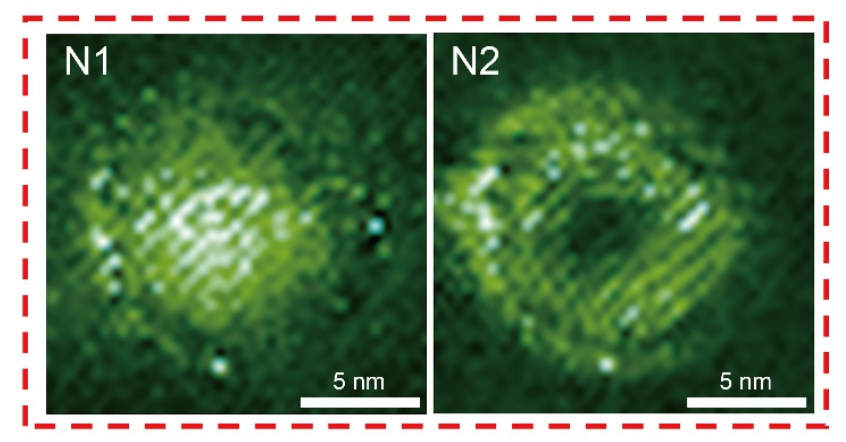

Fig. 1 The structures and general $\mathbf{d} I / \mathrm{d} V$ features of Graphene/WSe $\mathrm{We}_{2}$ heterostructure. a, A representative AFM image of the freshly mechanical exfoliated $\mathrm{WSe}_{2}$ sheet. Inset: the AFM image of a typical monolayer $\mathrm{WSe}_{2}$ island. $\mathbf{b}, \mathrm{A}$ STM image of a typical graphene/WSe 2 heterostructure

5 QD. The height of the GQD is $\sim 0.8 \mathrm{~nm}$ and the width of edge area of the GQD is $\sim 2.2 \mathrm{~nm}$. $\mathbf{c}$, The zoom-in image of the area in black dashed squares from panel b. Inset: the FFT of graphene/WSe 2 heterostructure. The bright spots in the white dotted circles represent the reciprocal lattice of graphene, the bright spots in the blue dotted circles represent the reciprocal lattice of $\mathrm{WSe}_{2}$, and 
the bright spots in the green dotted circles represent moire structure of the graphene/ $\mathrm{WSe}_{2}$ heterostructure. d, Schematic structure of the graphene/WSe ${ }_{2}$ heterostructure QD. e, The $\mathrm{d} I / \mathrm{d} V$ spectra taken inside [marked by dark green pentagram in b] and outside [marked by blue pentagram in $\mathbf{b}$ ] the GQD. $\mathbf{f}$, The $\mathrm{d} I / \mathrm{d} V$ maps with different energies [N1 and $\mathrm{N} 2$ marked in $\mathbf{e}]$ of 5 the GQD. 

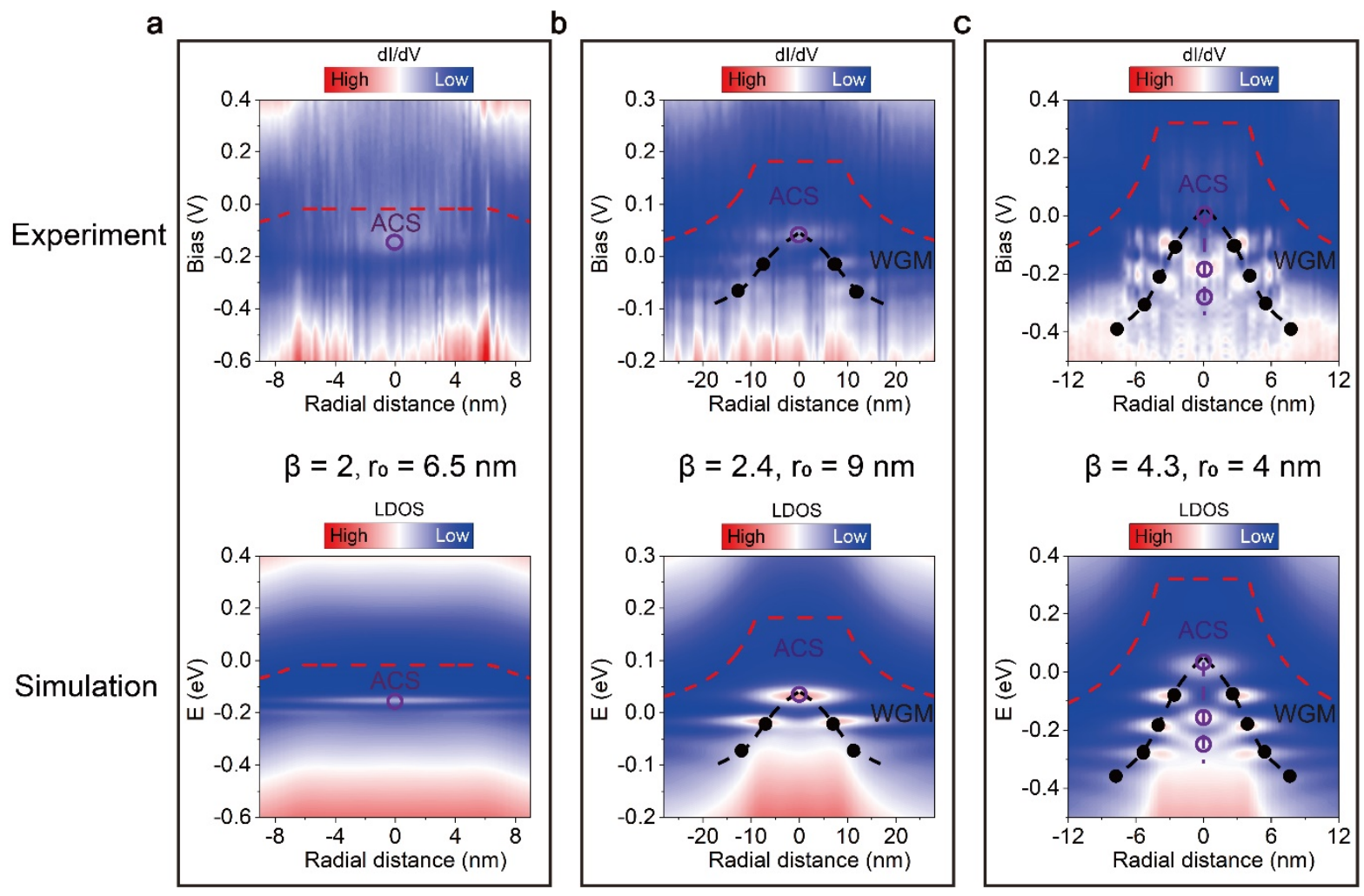

Fig. 2 Coexistence of WGMs confinement and ACSs in the GQDs. Top of a to $\mathbf{c}$, The radially $\mathrm{d} I / \mathrm{d} V$ spectroscopic maps of different GQDs. Bottom of a to $\mathbf{c}$, The calculated space-energy maps of the LDOS of different GQDs with different value of $\beta$ and $r_{0}$. The red dotted lines indicate Dirac

5 point energy. The black solid dots indicate the quasibound states via the WGM confinement, and the purple hollow dots indicate the ACSs. 


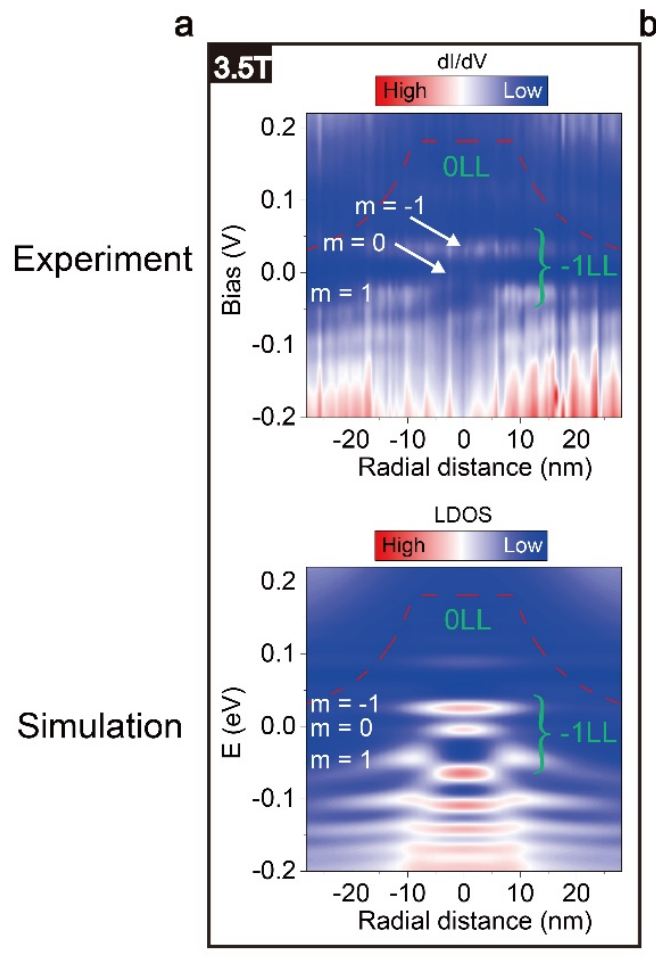

\section{b $6 T$}
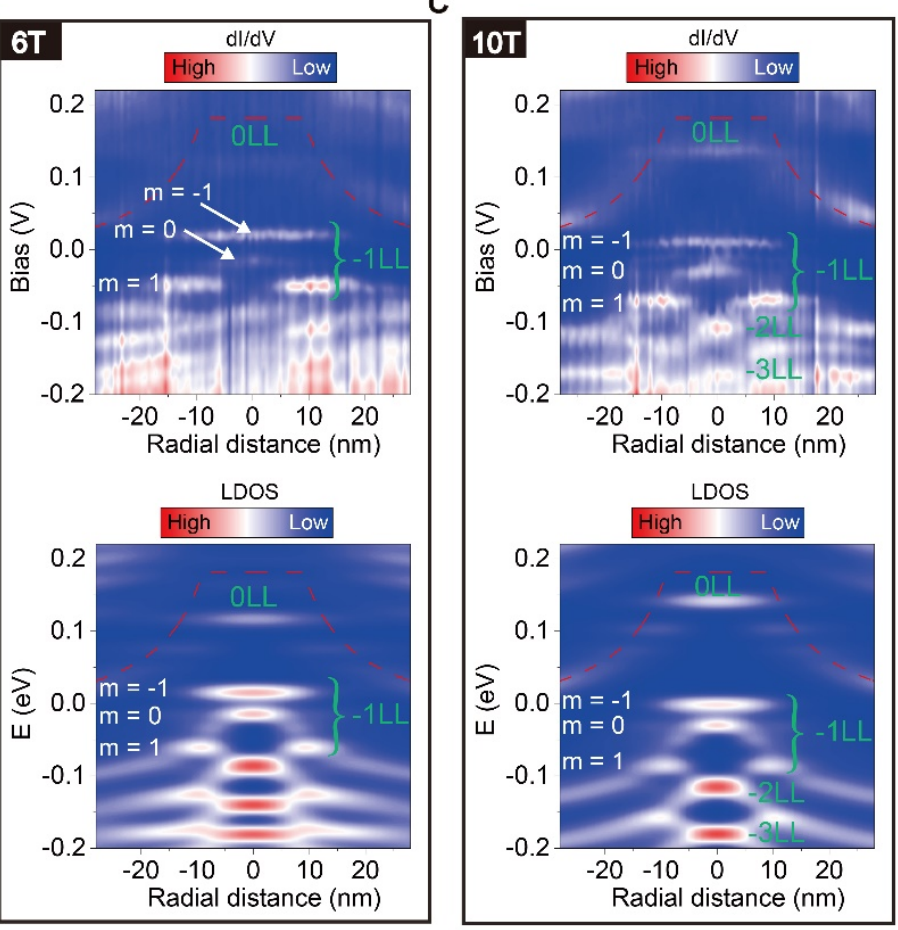

increasing $B$

Fig. 3 Lifting the orbital degeneracy of LLs in the GQD. Top panels of a to $\mathbf{c}$, the radially $\mathrm{d} I / \mathrm{d} V$ spectroscopic maps on the GQD $\left(\beta=2.4, r_{0}=9 \mathrm{~nm}\right)$ in the case of a series of magnetic fields. Bottom panels of a to $\mathbf{c}$, the calculated space-energy maps of the LDOS of the GQD with

5 different magnetic fields. The $m=-1, m=0$, and $m=1$ indicate the split orbital states of the -1 LL. The red dotted lines indicate Dirac point energy. 


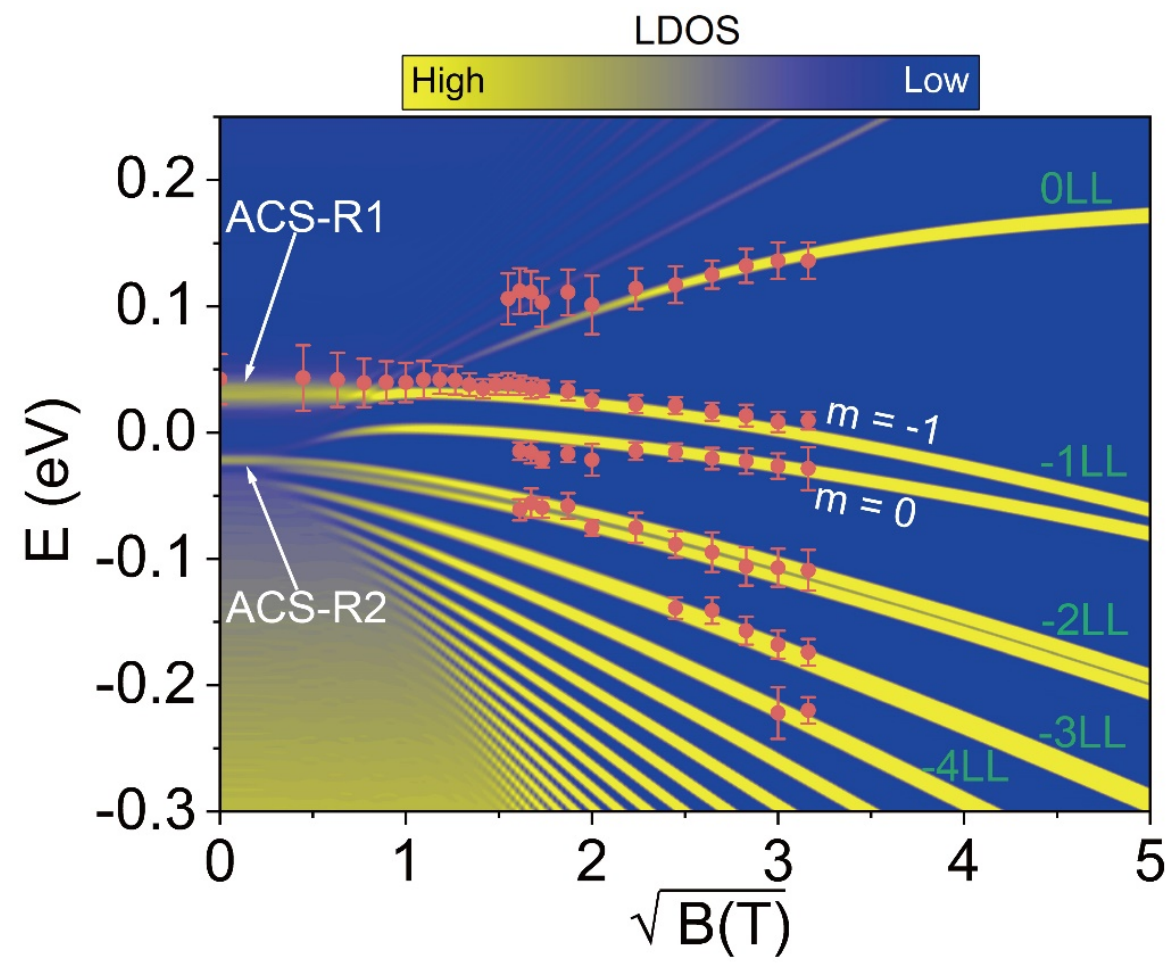

Fig. 4 Evolution from the ACSs to unusual LLs in the GQD. The measured LLs at the center of the GQD $\left(\beta=2.4, r_{0}=9 \mathrm{~nm}\right)$ as a function of the square root of the magnetic field $\sqrt{B}$. The experimental results are superimposed onto the calculated map of LDOS in the GQD with $\beta=2.4$

5 and $r_{0}=9 \mathrm{~nm}$. The ACS-R1 and ACS-R2 are two quasi-bound states due to atomic collapse resonance. The full width at half maximum of the peaks in the spectra was used to estimate the error bar in experiment (orange dots). 


\section{Supplementary Files}

This is a list of supplementary files associated with this preprint. Click to download.

- SupplementaryInformation.docx 